\title{
Investition oder Geldschneiderei? Nahrungsergänzungsmittel im Kleintiersektor
}

\author{
Cornelia Rückert
}

Auf dem Heimtierfuttermarkt werden zahlreiche Ergänzungsfuttermittel mit teils vielversprechenden Wirkungen angeboten. Ein Überblick über die Produkte, deren Wirkmechanismen und den Nutzen einer Supplementierung zur täglichen Futterration ermöglicht eine professionelle Empfehlung gegenüber dem Besitzer.

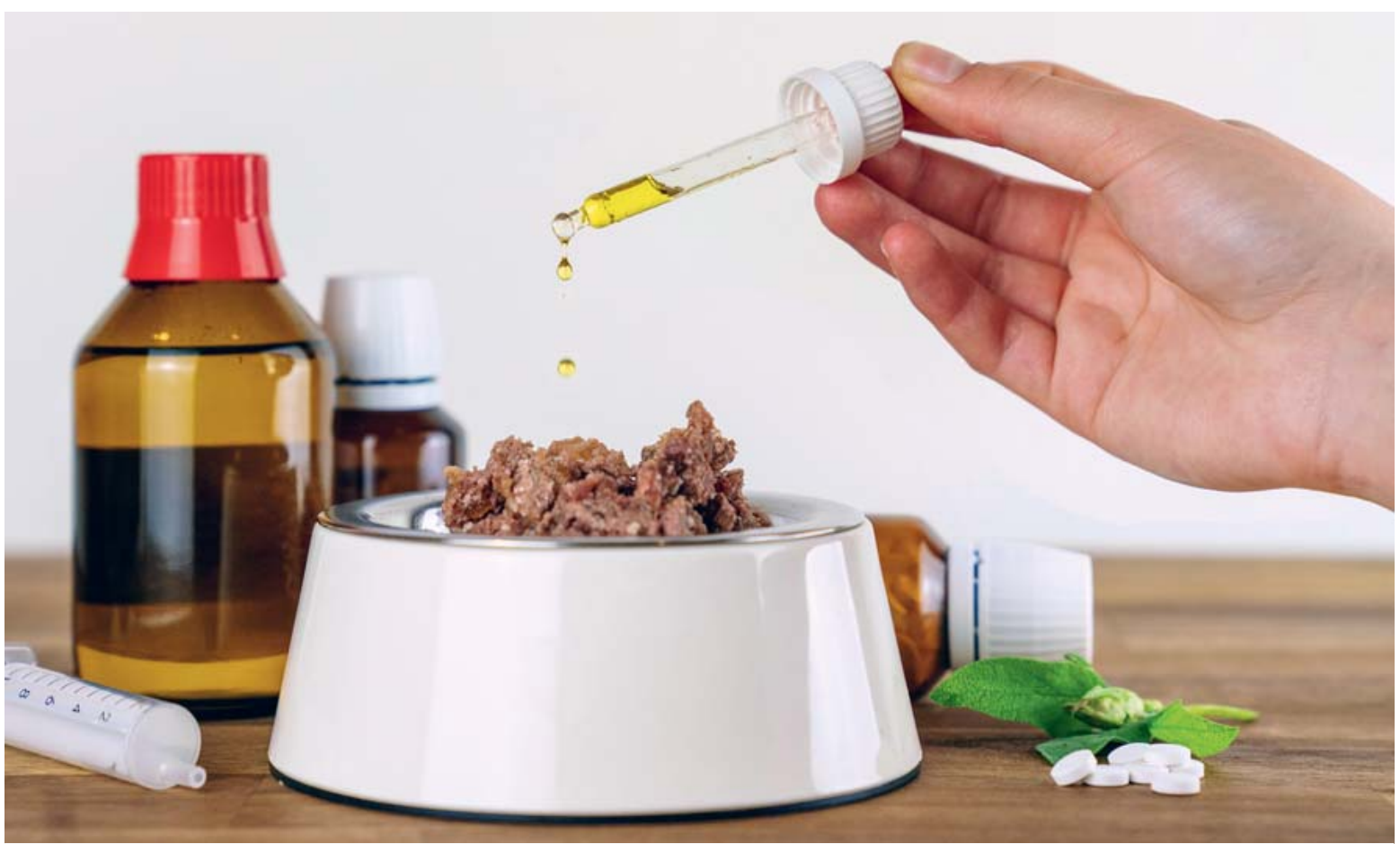

Quelle: Kirsten Oborny, Thieme Gruppe

\section{Bewegungsapparat}

\section{Gelatine-/Kollagenhydrolysat}

Bei der Zufütterung von Gelatinehydrolysat an Hunde mit chronischen arthrosebedingten Lahmheiten wurden positive Effekte auf den Lahmheitsgrad und die Schmerzhaftigkeit festgestellt. So stiegen unter einer täglichen Gabe von $20 \mathrm{~g}$ Gelatinehydrolysat/Tier die Blutplasmakonzentrationen der an der Kollagensynthese beteiligten Aminosäuren Glyzin, Prolin und Hydroxyprolin an. Gleich- zeitig konnte ein Abfall der Enzyme MMP-3 (Matrix-Metalloprotease 3) und bAP (knochenspezifische alkalische Phosphatase), die bei Arthritis- und Arthrosepatienten erhöht sind, verzeichnet werden. Daher wurde postuliert, dass die orale Gabe von Gelatinehydrolysat bei Hunden, die eine Prädisposition für degenerative Skeletterkrankungen haben, z. B. genetisch belastete Linien oder intensive sportliche Nutzung, sinnvoll sein kann.

Humanmedizinische Studien zum Behandlungserfolg nach oraler Einnahme bei degenerativen Cox- und Gon- 
arthrosen zeigten signifikante Besserungen gegenüber einer Kontrollgruppe. Auch beim Einsatz an 100 geriatrischen Patienten zeigten sich sowohl positive Effekte auf die Bewegungsumfänge der betroffenen Gelenke wie auch eine Reduzierung der Schmerzhaftigkeit. An Mäusen wurde nach oraler Verabreichung radioaktiv gekennzeichneten Gelatinehydrolysats eine Wiederfindbarkeit im Knorpel nachgewiesen. In vitro konnte nach Anreicherung mit Kollagenhydrolysat eine gesteigerte KollagenTyp-2-Synthese in Chondrozytenkulturen nachgewiesen werden.

\section{Bioaktive Kollagenpeptide}

Diese liefern Glyzin, Prolin und Hydroxyprolin, welche die Baueinheiten für Kollagen darstellen. Ein Beitrag zum Erhalt und sogar zu einer eventuellen Neubildung von Knorpelsubstanz wird daher aktuell diskutiert. In klinischen Studien zeigten Hunde mit chronischen Erkrankungen des Bewegungsapparats unter der oralen Gabe von täglich $20 \mathrm{~g}$ bioaktiver Kollagenpeptide eine signifikante Abnahme des Lahmheitsgrads und eine Zunahme der Bewegungsfreude. Jedoch muss auch hier beachtet werden, dass die Einschätzung teilweise dem Patientenbesitzer überlassen wurde. Die beobachteten positiven Effekte am Patienten gingen mit einer Abnahme von Matrix-Metalloprotease 3 (MMP-3) und einer Zunahme des Metallopeptidase-Inhibitor-1 (TIMP-1) im Plasma einher.

\section{Glykosaminoglykane}

Mittels von Glykosaminoglykanen wie Chondroitinsulfat will man eine Regeneration des hyalinen Gelenkknorpels durch die Syntheseleistung der Chondrozyten und der Synoviozyten mit Hyaluronsäure bewirken. Die Glykosaminoglykane besitzen negativ geladene Sulfatgruppen durch diese sollen die positiv geladenen Aminosäurengruppen der Enzymproteine (z.B. Metalloproteasen und lysosomale Enzyme) gebunden und somit gehemmt werden. In zahlreichen Studien wurde die Resorptionsrate und der klinische Effekt oral zugeführter Glukosaminoglykane untersucht, die Ergebnisse sind teils sehr divergent. Während mittels radioaktiver Detektionsmethoden sowohl eine Resorption aus dem Darm wie auch ein Einbau in den Gelenkknorpel nachgewiesen werden konnte, sind die klinischen Ergebnisse weniger einheitlich. Während auf der einen Seite in 75\% der Fälle von einer Besserung der klinischen Symptome gesprochen wird, zeigen andere Arbeiten bei > 50\% der Patientengruppe keinen signifikanten Effekt. Hierbei ist jedoch auch zu beachten, dass oftmals Feldstudien durchgeführt wurden und eine Einschätzung des Behandlungserfolgs dem Besitzer überlassen wurde. Meist wurde hier mit Dosierungen von 75$100 \mathrm{mg} /$ Tag für Glukosamin und $1000-1500 \mathrm{mg} /$ Tag für Chondroitinsulfat gearbeitet.

Auf dem Futtermittelmarkt werden verschiedenste Produkte aus der neuseeländischen Grünlippigen Muschel als Lieferant von Glykosaminoglykanen angeboten. Die
Konzentrationen der Glykosaminoglykane liegen hier jedoch durchgehend unterhalb der Nachweisgrenze. Nur Muschelextrakte enthielten Spuren von Glykosaminoglykanen, welche auch hier nicht im Rahmen einer klinisch relevanten Dosierung liegen.

Dosierung:

- Glukosamin: 75-100 mg/Tag

- Chondroitinsulfat: 1000-1500 mg/Tag

\section{Aminosäuren}

Hier ist - neben der Bedarfsdeckung mit essenziellen Aminosäuren, die bei einer durchschnittlichen Hunderation aber meist keine Probleme darstellt - vor allem die Kollagensynthese von Bedeutung. Hierfür werden schwefelhaltige Aminosäuren (Methionin und Cystein) sowie nicht essenzielle Aminosäuren (Prolin, Hydroxyprolin und Glyzin) benötigt. In einer Arbeit wurden bei Hunden mit chronischen Erkrankungen des Bewegungsapparats im Vergleich zu orthopädisch unauffälligen Hunden erniedrigte Plasmaspiegel an Glyzin, Prolin und Hydroxyprolin nachgewiesen.

Die Aminosäurenzusammensetzung im Blutplasma spiegelt auch die Aufnahme über das Nahrungsprotein wider. So kann bei Hunden mit Gelenkerkrankungen eine Minderversorgung mit bindegewebsassoziierten Proteinen nicht ausgeschlossen werden. Jedoch ist auch hier ein bisher nicht bekannter modifizierter Proteinkatabolismus bei den betroffenen Hunden nicht auszuschließen.

\section{Omega-3-Fettsäuren}

Omega-3-Fettsäuren haben einen nachweislich antiinflammatorischen Effekt. Die Einlagerung von Eicosapentaensäure (EPA) in die Zellmembran wird forciert, und durch den Metabolismus von EPA werden weniger entzündungsfördernde Botenstoffe gebildet. In mehreren Versuchen zeigte die diätetische Zulage von Omega3-Fettsäuren eine deutliche Verbesserung des Gangbilds und der Schmerzhaftigkeit arthrotischer Patienten.

- Dosierung: 1 g EPA + DHA (Docosahexaensäure)/10 kg

\section{Methylsulfonylmethan}

Methylsulfonylmethan (MSM) dient als Quelle für organische Schwefelverbindungen. Zusätzlich wird eine antiinflammatorische und antioxidative Wirkung postuliert. In einer humanmedizinischen Arbeit wurde zudem eine stimulierende Wirkung auf die Osteoblastendifferenzierung nachgewiesen.

Die veterinärmedizinischen Studien sind bisher wenig aussagekräftig. In einer Studie an 24 geriatrischen Pferden wurde ein Kombinationspräparat aus Glukosamin, Chondroitinsulfat und Methylsulfonylmethan verabreicht, sodass die beobachteten positiven Effekte nicht allein einer der Ausgangssubstanzen zugeschrieben werden können. Gleiches gilt für eine Studie an Ratten. Für Hund 
oder Katze liegen derzeit keine wissenschaftlichen Studien zur Wirksamkeit vor.

\section{Hyaluronsäure}

Diese soll hier nur am Rande erwähnt werden, da bisher keinerlei Effekte nach oraler Verabreichung beobachtet werden konnten. Einzig die intraartikuläre Injektion wird in der Human- sowie Veterinärmedizin beschrieben und teils kontrovers diskutiert.

\section{Risikofaktor Übergewicht?}

Zu all den genannten prophylaktischen und therapeutischen Möglichkeiten der Arthrosebekämpfung sollte eines nicht außer Acht gelassen werden: Adipositas ist einer der Hauptrisikofaktoren für die Entstehung von arthrotischen Veränderungen. Zudem können bereits bestehende klinische Beschwerden bei Arthrose durch die Verringerung von Übergewicht signifikant verbessert werden. Daher können die genannten Ergänzungsfuttermittel nur eine ggf. sinnvolle Unterstützung der beiden primären Therapiepunkte „Gewichtskontrolle bzw. -reduktion“ und „angepasstes Bewegungsmanagement“ darstellen. Dies auch in dieser Form mit dem Patientenbesitzer zu kommunizieren, ist aus Autorensicht eine dringlichere Aufgabe als die Empfehlung zur Fütterung verschiedener kommerzieller Ergänzungsfuttermittel.

\section{ZWISCHENFAZIT BEWEGUNGSAPPARAT \\ Reduktion von Übergewicht bzw. Erhalt des Ideal- gewichts - in Kombination mit gezieltem Muskelauf- bau und einer physiotherapeutischen Betreuung des Patienten - sollten an 1. Stelle der Prioritätenliste stehen. \\ Zusätzlich dazu kann folgende ernährungsphysiolo- gische Unterstützung empfohlen werden: \\ - Supplementierung bioaktiver Kollagenpeptide oder von Gelatinehydrolysat jeweils mit einer Dosierung von $20 \mathrm{~g} /$ Tier/Tag \\ - Ergänzung mit Omega-3-Fettsäuren, um vor allem akute Entzündungsschübe zu verhindern bzw. abzumildern: $1 \mathrm{~g}$ EPA + DHA/10 kg/Tag}

\section{Haut und Fell}

\section{Bierhefe}

Aufgrund des hohen Gehalts an B-Vitaminen (v.a. $B_{1}$, aber auch $B_{2}, B_{3}, B_{5}, B_{6}, B_{9}, B_{12}$ ) eignet sich Bierhefe zur Supplementierung bei Patienten, die stumpfes, glanzloses Fell aufweisen. Häufig zeigen die betreffenden Patienten schon nach kurzer Zeit eine deutliche Besserung der Fellqualität. Vor allem zur Unterstützung im Fellwechsel hat sich eine Bierhefesupplementierung bewährt.

- Dosierungsempfehlung: 1 g/10 kg
Zu Beginn der Gabe sollte hier jedoch mit einer niedrigeren Dosierung begonnen werden, da es unter Umständen durch die Verschiebung der intestinalen Mikrobiota anfänglich zu Meteorismus und/oder einer Verschlechterung der Kotkonsistenz kommen kann.

\section{Merke}

Vorsicht ist bei Hunden und Katzen geboten, die aufgrund von Juckreiz unbekannter Genese vorstellig werden.

Sollte hierfür ursächlich ein allergisches Geschehen zugrunde liegen, könnte die Problematik durch das allergene Potenzial der Bierhefe verstärkt werden.

\section{Biotin}

Biotin ist an der Bildung von Keratin beteiligt und somit ein essenzieller Baustein für Haut, Fell und Krallen. Für den Hund wird ein Tagesbedarf von $2 \mathrm{mg} / \mathrm{kg}$ angesetzt - wird dieser dauerhaft unterschritten, äußert sich das in einem Stumpfwerden des Felles, in Haarausfall, Juckreiz und damit verbundenen Hauteffloreszenzen. Wird der Biotinbedarf nicht über die tägliche Futterration gedeckt, ist eine Supplementierung als Monopräparat sinnvoll. Häufig wird die Fütterung roher Eier empfohlen. Hierbei sollte jedoch beachtet werden, dass nur im Eigelb Biotin enthalten ist. Das Eiklar hingegen beinhaltet Avidin, welches Biotin bindet und damit die Verfügbarkeit stark einschränkt. Daher sollte, wenn überhaupt, nur das rohe Eigelb verfüttert werden. Auch auf die Infektionsgefahr mit Salmonellen durch die Zufütterung rohen Eimaterials sei an dieser Stelle hingewiesen.

\section{Öle/Fettsäuren}

Vor allem bei Patienten mit atopischer Dermatitis, einer immunvermittelten Schädigung des Säureschutzmantels und damit der Hautbarriere, hat sich der Einsatz essenzieller Fettsäuren, insbesondere Fischölen, gut bewährt. Die diätetische Zufuhr essenzieller Fettsäuren beeinflusst die Membranfluidität und die damit verbundenen Prozesse wie Enzymaktivitäten und Rezeptorfunktionen. Zudem beeinflussen sie die allergische Reaktion durch die Modulation der Prostaglandin- und LeukotrienProduktion. In zahlreichen Studien konnte ein Effekt der Zulage von Linol-, $\alpha$-Linolen-, $\gamma$-Linol-, Eicosapentaen(EPA) und Docosahexaensäure (DHA) gezeigt werden. Die Patienten hatten eine verbesserte Haarqualität, die Hautbarriere wurde gestärkt, es kam zu einer Reduktion des transepidermalen Wasserverlusts, und die Dosierung zusätzlich eingesetzter Medikamente, wie Glukokortikoide und Ciclosporin A, konnte reduziert werden.

- Dosierungsempfehlung für die relevanten Fettsäuren: mindestens $50 \mathrm{mg} / \mathrm{kg}$

Als Lieferant von Linol- und $\alpha$-Linolensäure dienen pflanzliche Öle wie Leinöl. EPA und DHA sind vor allem in marinen Ölen in hohen Mengen enthalten (Fett von Meeresfischen wie Lachs, Hering oder Makrele). 


\section{ZWISCHENFAZIT HAUT UND FELL}

Die Qualität von Haut und Fell lässt sich sehr gut über Ergänzungsfuttermittel beeinflussen - vorausgesetzt, es sind keine anderweitigen Grunderkrankungen oder mangelhafte Pflege ursächlich.

Hierzu kann folgendes Supplementierungsschema angewendet werden:

- Bierhefe: $~ 1 \mathrm{~g} / 10 \mathrm{~kg}$ (cave: Allergiker)

- Biotin: Bedarf 2 mg/kg (Überdosierung ist unbedenklich, da Biotin als Vitamin der B-Gruppe wasserunlöslich ist)

- Omega-3-Fettsäuren: 50 mg/kg (Kombination mariner und pflanzlicher Öle)

\section{Zahnpflege}

\section{Polyphosphate}

An der Ausbildung von Zahnstein ist vor allem im Speichel befindliches Kalzium beteiligt. Dieses wird durch die mikrokristallinen Polyphosphate (z. B. Hexametaphosphat) gebunden, welche meist in die Außenhüllen der Futterkroketten eingearbeitet sind. Dadurch wird verhindert, dass sich die Kalziumverbindungen an die Zähne anheften und Zahnstein ausbilden. Zudem bewirken die Mikrokristalle einen gewissen Abriebeffekt des Plaques an den Kauflächen. Durch den langsamen Abbau der Polyphosphate bleibt die Wirkung auch über die eigentliche Dauer der Futteraufnahme hinaus bestehen.

\section{Faserstoffe}

Spezielle Fasern können helfen, einen mechanischen Reinigungseffekt auf die Zähne auszuwirken. Das Krokettenmaterial ist dabei so konzipiert, dass diese beim Draufbeißen nicht zerbröseln, sodass die Zähne tiefer in die Futterstücke eindringen, was einen mechanischen Effekt am Zahnhals erzielt. Die meisten Parodontopathien entstehen jedoch am Zahnfleischsaum, dieser wird allein durch diese diätetischen Fasern meist nicht ausreichend gereinigt. Zudem gibt es gerade unter Hunden Individuen, die auch größere Futterbrocken ohne zu Kauen herabschlucken, sodass hier der mechanische Reinigungseffekt entfällt.

\section{ZWISCHENFAZIT ZAHNPFLEGE}

Bei Patienten, die verstärkt zu Zahnsteinbildung neigen, sollte der Besitzer eine regelmäßige mechanische Zahnreinigung (Zahnbürste, „Putzfinger“ o. ä.) durchführen. Diese kann durch geeignete Präparate aus dem Handel, welche die oben genannten Polyphosphate und Faserstoffe enthalten, ergänzt werden.

\section{Verdauung}

\section{Probiotika}

Hierbei handelt es sich um „lebende Mikroorganismen“, zumeist Laktobazillen und Enterokokken, welche oral aufgenommen werden und das physiologische Mikrobiom im Darm unterstützen sollen. Der Nutzen ist jedoch umstritten. Die Größenordnungen der in klinischen Versuchen beobachteten Veränderungen sind meist vernachlässigbar.

\section{Präbiotische Fasern}

Diese Fasern sollen den im Darm befindlichen Mikroorganismen als Nahrung dienen. Sie enthalten Zuckeroder Faserverbindungen, die nicht körpereigen abbaubar sind. Aufgrund dieser Tatsache kommen sie nahezu unverändert im Dickdarm an und werden durch die dort ansässigen Bakterien fermentiert.

Dies hat 2 positive Effekte:

- Zum einen wird die physiologische (also „erwünschte“) Mikrobiota im Darm gefördert,

- zum anderen entstehen durch den Fermentationsprozess kurzkettige Fettsäuren, v. a. Butyrat.

Butyrat hat nachgewiesenermaßen einen positiven Effekt auf die Regeneration und das Wachstum der Darmzotten, da es für diese als Energielieferant dient. Zudem entsteht durch die kurzkettigen Fettsäuren ein leicht saures Darmmilieu, welches von den meisten pathogenen Bakterien nicht gut toleriert wird.

Zu den Präbiotika zählt z. B. Inulin, ein Polysaccharid aus Fruktoseeinheiten, welches in Chicoréewurzeln oder Topinamburknollen enthalten ist. Ebenfalls häufigen Einsatz findet hierbei Pektin, welches sich in Äpfeln, Möhren oder Rübenschnitzeln findet. Hier sollten beim Einsatz jedoch auch die recht hohen Anteile körpereigen verwertbarer Zucker beachtet werden.

Werden die beschriebenen Fasern eingesetzt, sollte der Patient immer langsam daran adaptiert werden, damit sich auch die intestinalen Bakterienstämme langsam umstellen können. Sonst kann es in der Anfangszeit schnell zu Flatulenzen oder einer weicheren Kotkonsistenz kommen. Zudem regen die Fasern die Darmmotilität an.

Häufig fällt auf, dass Hunde, welche überwiegend mit Trockenfutter ernährt werden, eine bessere Kotkonsistenz aufweisen als ihre Artgenossen, die überwiegend Feuchtfutter erhalten. Das mag zum einen daran liegen, dass Feuchtfutter häufig hohe Mengen an Protein mit schlechterer Verdaulichkeit (Innereien und Schlachtabfälle) beinhalten, welche unverdaut im Dickdarm ankommen und dort z. B. von Clostridien verstoffwechselt werden. Andererseits beinhalten Trockenfutter öfter und hö- 
here Mengen an pflanzlichen Anteilen, deren Faserfraktionen hier einen präbiotischen Effekt aufweisen. In 2 Studien konnte gezeigt werden, dass mit Trockenfutter gefütterte Hunde ein vergleichbares fäkales Mikrobenprofil aufwiesen wie Hunde, die Präbiotika erhielten.

\section{ZWISCHENFAZIT VERDAUUNG}

Chronische Verdauungsprobleme, insbesondere Flatulenzen oder Diarrhö, sind nach Ausschluss anderer Ursachen, wie infektiöse Ursachen oder Unverträglichkeiten, sehr gut über eine angepasste Ernährung zu beeinflussen.

Neben einer Rationsüberprüfung bzw. -anpassung, die u.a. den Fettgehalt und den Gehalt sowie die Verdaulichkeit eingesetzter Proteinquellen sowie der Kohlenhydratträger berücksichtigt, ist der Einsatz präbiotischer Substanzen ratsam.

Hierbei können als Präbiotika zum Einsatz kommen (bitte immer langsame Adaptation beachten):

- Pektine (Apfel, Möhre etc.)

- Fructooligosaccharide

- Inulin (z. B. aus Topinambur)

- Laktulose

Eine genaue Dosierungsangabe ist für die meisten Substanzen nicht sinnvoll, da diese stark vom Alter, dem Gesundheitszustand sowie der bisherigen mikrobiellen Ausstattung im Dickdarm abhängen.

Meist wird von einer Endzielmenge von $0,5 \mathrm{~g} / \mathrm{kg}$ ausgegangen, diese muss jedoch über einen Zeitraum von 1-2 Wochen langsam anvisiert werden. Beginnen sollte man mit Tagesdosen von 0,5 (Hunde bis $10 \mathrm{~kg}$ ), $1 \mathrm{~g}$ (Hunde bis $30 \mathrm{~kg}$ ) oder $2 \mathrm{~g}$ (Hunde über $30 \mathrm{~kg}$ ).

Bei chronischer Diarrhö oder weicher Kotqualität können auch wasserbindende und somit kotfestigende Stoffe gefüttert werden, z. B. Weizenkleie oder Flohsamenschalen.

\section{Verhaltensmodulation}

\section{Casozepin}

Dieses wird unter verschiedenen Handelsnamen mit einer anxiolytischen Wirkung beworben. $\alpha$-Casozepin ist ein bioaktives Peptid, welches aus Milch durch tryptische Hydrolyse gewonnen wird. Es besitzt eine spezifische Bindungsstelle auf dem Rezeptor für $\mathrm{Y}$-Aminobuttersäure und potenziert damit deren hemmenden Effekt. In einer Studie wurde die Wirkung mit der von Selegilin verglichen. Hier zeigte sich eine vergleichbare anxiolytische Wirkung zwischen den beiden Substanzen (Dosierung Casozepin: $15 \mathrm{mg} / \mathrm{kg}$ ). Es sollte jedoch beachtet werden, dass mit einem Wirkungseintritt erst nach einer Behandlungsdauer von 1-2 Wochen zu rechnen ist. 


\section{L-Tryptophan}

Diese Aminosäure ist die Vorläuferstufe des Neurotransmitters Serotonin. Erhöhte Tryptophan-Spiegel im Gehirn bedingen eine gesteigerte Serotoninfreisetzung. Bei der Überwindung der Blut-Hirn-Schranke steht Tryptophan hierbei in Konkurrenz mit den langkettigen neutralen Aminosäuren Tyrosin, Phenylalanin, Valin, Leucin und Isoleucin. Alle benutzen dasselbe Carrier-System. Positive verhaltensändernde Wirkungen, v.a. im Hinblick auf Angstverhalten, wurden mit einer Diät erzielt, welche ein erhöhtes Verhältnis von Tryptophan zu den langkettigen neutralen Aminosäuren hatte.

\section{ZWISCHENFAZIT VERHALTENSMODULATION \\ Neben einer an den Patienten angepassten Verhal- tenstherapie bzw. professionellem Training kann die Gabe von Casozepin und L-Tryptophan eine sinnvolle Ergänzung für stark gestresste oder ängstliche Tiere sein. Eine Kombination der Gabe von Casozepin und L-Tryptophan kann daher angeraten werden. Dosierung: \\ - Casozepin: $15 \mathrm{mg} / \mathrm{kg}$ \\ - L-Tryptophan: $2,5 \mathrm{mg} / \mathrm{kg}$}

\section{Mineralfuttermittel}

Vor einer Supplementierung von Mineralstoffen sollte die bisher verfütterte Ration kritisch überprüft werden. Handelt es sich hierbei um ein kommerzielles Alleinfuttermittel, ist eine Mineralstoffergänzung meist nicht notwendig bzw. kann sogar kontrainduziert sein. Eine überhöhte Aufnahme an Mengenelementen kann zur Ausbildung von Harnkonkrementen oder im Fall einer Kalzium- oder Phosphorfehlversorgung beim Junghund beispielsweise zu Wachstumsstörungen aufgrund einer Fehlmineralisation des Skelettsystems führen. Ähnlich verhält es sich mit überhöhten Spurenelementaufnahmen. Auch hier ist mit zahlreichen Wechselwirkungen und Folgen einer chronisch bedarfsüberschreitenden Aufnahme zu rechnen, die an dieser Stelle jedoch nicht alle genannt werden können.

Anders verhält es sich, wenn der Patientenbesitzer selbst zusammengestellte Rationen verfüttert (gekocht oder BARF).

Bei gekochten oder BARF-Rationen sind zumeist gezielte Ergänzungen im Bereich der Mengen- und Spurenelemente wie auch Vitamine notwendig.

Diesen Ergänzungen sollte jedoch immer eine Kalkulation der bisher angebotenen Ration vorangehen, um Nährstoffdefizite aufzudecken und diese Lücken gezielt mit Ergänzungsfuttermitteln aufzufüllen.
}

\section{TAKE HOME}

\section{Bewegungsapparat}

- Ziel: Erhalt des Idealgewichts in Kombination mit Muskelaufbau und einer physiotherapeutischen Behandlung

- ggf. Reduktion von Übergewicht

- ernährungsphysiologische Supplementierung empfehlenswert:

- bioaktive Kollagenpeptide oder Gelatinehydrolysat: $20 \mathrm{~g} / \mathrm{Tier} / \mathrm{Tag}$

- Omega-3-Fettsäuren: $1 \mathrm{~g}$ EPA + DHA/ $10 \mathrm{~kg} / \mathrm{Tag}$

\section{Haut und Fell}

- Ausschluss anderweitiger Grunderkrankungen oder mangelhafter Pflege

- ernährungsphysiologische Supplementierung empfehlenswert:

- Bierhefe: $1 \mathrm{~g} / 10 \mathrm{~kg}$ (cave: Allergiker)

- Biotin: Bedarf $2 \mathrm{mg} / \mathrm{kg}$

- Omega-3-Fettsäuren: 50 mg/kg (Kombination mariner und pflanzlicher Öle)

\section{Zahnpflege}

- regelmäßige mechanische Zahnreinigung mit Zahnbürste oder „Putzfinger“

- ggf. ernährungsphysiologische Supplementierung mit Polyphosphaten und Faserstoffen empfehlenswert

\section{Verdauung}

- chronische Verdauungsprobleme gut über eine angepasste Ernährung beeinflussbar

- Rationsüberprüfung bzw. -anpassung

- Einsatz präbiotischer Substanzen (Pektine, Fructooligosaccharide, Inulin, Laktulose) empfehlenswert

- bei chronischer Diarrhö oder weicher Kotqualität auch wasserbindende und somit kotfestigende Stoffe (z. B. Weizenkleie oder Flohsamenschalen) empfehlenswert

\section{Verhaltensmodulation}

- Verhaltenstherapie bzw. professionelles Training

- Supplementierung mit Casozepin (15 mg/kg) und L-Tryptophan $(2,5 \mathrm{mg} / \mathrm{kg})$ für stark gestresste oder ängstliche Tiere empfehlenswert

\section{Mineralfuttermittel}

- Indikation prüfen

- bei kommerziellem Alleinfuttermittel ggf. kontraindiziert

- bei selbst zusammengestellter Ration gezielte Ergänzungen mit Mengen- und Spurenelementen sowie Vitaminen empfehlenswert 


\section{Fazit}

Der Markt bietet ein schier unüberschaubares Angebot an verschiedensten Supplementen, die mit einem gesundheitsfördernden Effekt beworben werden. Vielfach halten diese Produkte jedoch nicht, was sie versprechen. Seien es zu geringe Wirkstoffkonzentrationen oder nicht klinisch belegte Wirksamkeiten. Daher ist vor einem gezielten Einsatz die Herstellerbewerbung kritisch zu hinterfragen und eventuell auf nicht diätetische Konzepte zurückzugreifen.

INFO

Hinweise zur Tierärztlichen Ernährungsberatung der Autorin finden Sie unter:

www.lkvsachsen.de/service/fuetterung

Korrespondenzadresse

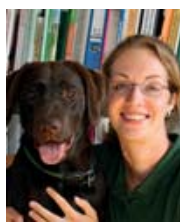

Dr. Cornelia Rückert

Fachtierärztin für Tierernährung und Diätetik Landwirtschaftliche Kommunikations- und Servicegesellschaft $\mathrm{mbH}$ August-Bebel-Str. 6 09577 Niederwiesa cornelia.rueckert@lks-mbh.com

\section{Literatur}

[1] Apanavicius C], Powell KL, Vester B. M et al. Fructan supplementation and infection affect food intake, fever, and epithelial sloughing from Salmonella challenge in weanling puppies. J Nutr 2007; 137: 1923-1930

[2] Beata C, Beaumont-Graff E, Coll V et al. Effect of alpha-casozepine (Zyklene) on anxiety in cats. J Vet Behav 2007; 2: 40-46

[3] Beata C, Beaumont-Graff E, Diaz C et al. Effects of alpha-casozepine (Zyklene) versus selegiline hydrochloride (Selgian, Anipryl) on anxiety disorders in dogs. J Vet Behav 2007; 2: 175-183

[4] Canapp SO, McLaughlin Jr RM, Hoskinson JJ et al. Scintigraphic evaluation of dogs with acute synovitis after treatment with glucosamine hydrochloride and chondroitin sulfate. Am J Vet Res 1999; 60: 1552-1557

[5] Hennet P, Servet E, Soulard Y et al. Effect of pellet food size and polyphosphates in preventing calculus accumulation in dogs. J Vet Den 2007; 24 (4): 236-239

[6] lams. Diätetisches Management von Zahnerkrankungen bei Hund und Katze. "News" Veterinärmed Magazin der The lams Company 2002; II: 2-6

[7] Jensen J, LoganE, Finney $O$ et al. Reduction in accumulation of plaque, stain, and calculus in dogs by dietary means. J Vet Dent 1995; 12 (4): 161-163

[8] Johnson RB, Cox ER, Lepine AJ. Recent cental advances for companion animals through dietary means. Orlando, Florida: The North American Veterinary Conference; 2002

[9] Jung S. Sind Glykosaminoglykane für den therapeutischen Effekt des Muschelextrakts verantwortlich? Tierärztl Prax 1998; 1: $17-20$ 
[10] Kavanagh K, Geldermann D. Oral glycosaminoglycans: a survey of responses. Aust Vet J 1999; 77 (4): 220-221

[11] Korthäuer W, de la Torre J. Behandlung deformierender Arthropathien beim Diensthund mit einem neuen Glykosaminoglykanpräparat. Kleintierprax 1992; 7: 467-478

[12] Lepine AJ, Murray SM, Cox ER. Clinical investigation of dental diet efficacy in the senior dog. Kyoto, Japan: $8^{\text {th }}$ World Veterinary Dental Congress; 2003

[13] Moreau M, Troncy E, Del Castilllo JRE et al. Effects of feeding a high omega-3 fatty acids diet in dogs with naturally occurring osteoarthritis. J Anim Physiol Anim Nutr 2013; 97 (5): 830837

[14] Müller MR, Linek M, Löwenstein C et al. Evaluation of cyclosporine-sparing effects of polyunsaturated fatty acids in the treatment of canine atopic dermatitis. Vet J 2016; 210: 77-81

[15] Oesser S, Seifert J. Stimulation of type II collagen biosynthesis and secretion in bovine chondrocytes cultured with degraded collagen. Cell Tissue Res 2003; 311 (3): 393-399

[16] Oesser S, Adam M, Babel W et al. Oral administration of (14)C labeled gelatin hydrolysate leads to an accumulation of radioactivity in cartilage of mice (C57/BL). J Nutr 1999; 129 (10): 1891-1895

[17] Olivry T, DeBoer DJ, Favrot C et al. Treatment of canine atopic dermatitis: 2010 clinical practice guidelines from the International Task Force on Canine Atopic Dermatitis. Vet Dermatol 2010; 21 (3): 233-248

[18] Rosh JK, Cross AR, Renberg WA et al. Evaluation of the effects of dietary supplementation with fish oil omega-3 fatty acids on weight bearing in dogs with osteoarthritis. J Am Vet Med Assoc 2010; 236 (1): 67-73

[19] Saevik BK, Bergvall K, Holm BR et al. A randomized, controlled study to evaluate the steroid sparing effect of essential fatty acid supplementation in the treatment of canine atopic dermatitis. Vet Dermatol 2004; 15 (3): 137-145

[20] Setnikar I, Giacchetti C, Zanolo G. Pharmakokinetics of glucosamine in the dog and man. Drug Res 1986; 36 (1): 729 735

[21] Slowinski J, Petrone DM, Battista G et al. Clinical comparison of two tartar control dentifrices: a twelfe week study. J Clin Dent 1998; 9 (4): 101-104

[22] Verlinden A, Hesta M, Hermans JM et al. The effects of inulin supplementation of diets with or without hydrolysed protein sources on digestibility, faecal characteristics, haematology and immunoglobulins in dogs. Br J Nutr 2006; 96 (5): 936-944

[23] Weide N. Der Einsatz von Gelatinehydrolysat bei klinisch-orthopädisch gesunden Hunden und Hunden mit chronischen Erkrankungen des Bewegungsapparats [Disseration]. Hannover: Tierärztliche Hochschule Hannover; 2004

\section{Bibliografie}

DOI https://doi.org/10.1055/a-1121-5278

Kleintier konkret 2020; 23: 14-22

(c) Georg Thieme Verlag KG Stuttgart · New York ISSN 1434-9132 